\title{
Minicomputer presentation of speech stimuli
}

\author{
JAMES L. KNIGHT, JR. \\ Purdue University, West Lafayette, Indiana 47907
}

\begin{abstract}
A brief introduction to minicomputer control of speech stimuli is presented. Several techniques that have proven useful in this area are described.
\end{abstract}

Because the typical laboratory minicomputer possesses a rather limited memory capacity, it would appear to be unsuited for generating speech stimuli, especially natural speech segments as long as entire words or sentences. Yet, over the past few years the minicomputer has become an increasingly popular laboratory tool for this purpose. The purpose of this paper is to provide an introduction to this application of minicomputers and to present a number of useful techniques that have evolved in this area. The emphasis will be on the techniques themselves, rather than on specific hardware and software implementations.

As a method for generating and manipulating speech stimuli, the computer offers some distinct advantages, for example, timing accuracies on the order of microseconds. This may be particularly significant in studies involving synchronization of speech channels as in dichotic listening. The computer also allows greater independence in manipulation of speech parameters than otherwise possible. For example, an experimenter can vary interword intervals without changing the physical characteristics of the words themselves. Other speech parameters easily manipulated by computer include word duration, amplitude, and onset/offset characteristics.

Finally, the laboratory minicomputer offers one more advantage of paramount importance: the ability to generate and control speech stimuli on-line. Although large computer systems afford most of the advantages mentioned above, it is often not possible to dedicate such systems to on-line experiment control. The minicomputer is ideal for this purpose. Largely, this possibility of on-line minicomputer speech control has not been utilized. Most often, the minicomputer has been used off-line to generate audio tapes that are later played back to subjects via a tape recorder. In this way the minicomputer serves as a low-cost substitute for larger computers but does not display its real potential. Many possibilities (including on-line interactive manipulation of speech stimuli, accurate response timing from

This project was supported in part by Grant MH 26302 from the National Institute of Mental Health. Requests for reprints should be sent to James L. Knight, Jr., Department of Psychological Sciences, Purdue University, West Lafayette, Indiana 47907. various speech features, and manipulation of speech feedback) remain unexplored.

In minicomputer speech generation, two general approaches have been taken. In one, the computer is used to control a peripheral speech synthesizer (e.g., Cohen \& Massaro, 1976). The synthesizer has a builtin repertoire of phonemes. The computer simply specifies which phonemes should be presented, as well as some timing and amplitude characteristics of individual phonemes. This approach is most useful in applications involving presentation of only a few phonemes. For word generation, this approach yields somewhat unnatural-sounding speech. Also, some speech characteristics such as accent, emphasis, and emotional quality may be difficult to obtain. Finally, a separate synthesizer would be needed for each separate speech channel to be presented.

In the second approach to minicomputer speech control (Knight \& Kantowitz, 1973; Poltrock \& Mathews, 1976), a digitized representation of an analog natural-speech signal is first stored in the computer memory. This digitized representation consists of a sequence of samples of the analog speech amplitude at successive, closely spaced intervals. For good quality speech the analog speech signal must be sampled at least 6,000 times/sec, but preferably at $10,000-15,000$ times/ sec. Thus, if speech stimuli exceed a $1 / 4 \mathrm{sec}$ or so, storage of all the digitized samples becomes a serious problem. In fact, this is the main limitation in using minicomputers in this area. Once a digitized representation has been obtained, it may be processed to control word duration, amplitude, etc. At playback the successive samples are presented via digital-to-analog converter (DAC) output channels. This second approach produces natural-sounding speech and is well suited for presenting entire words or word sequences. It is not, however, well suited to the manipulation of individual phonemes, since these are difficult to extract from natural-speech inputs. The remainder of this paper will be limited to the second approach to minicomputer speech presentation; there are more minicomputers than speech synthesizers in psychology laboratories.

\section{EQUIPMENT REQUIREMENTS}

Equipment requirements naturally depend upon the 
complexity of the speech stimuli one wishes to generate. The minimal system is a $4 \mathrm{~K}$ minicomputer with at least one analog-to-digital converter (ADC) for speech input and one DAC output channel. Some form of mass data storage (magnetic tape or disk) is also needed. With such a minimal system, presentation of word sequences, with presentation rate limited by the time needed to access words from mass storage, is possible. Word durations up to $1 \mathrm{sec}$ or so would be possible. A more complex system affords three primary advantages: (1) decreased interword intervals and thus faster presentation rates, (2) simultaneous presentation of more than one word, as in dichotic listening, and (3) enhanced ability to perform other on-line aspects of experiment control, such as response monitoring, in addition to speech generation. A very sophisticated system might involve a minicomputer with $12 \mathrm{~K}$ of memory capacity, at least two ADCs and two DACs, and a disk for high-speed mass storage. With such a system, dichotic word sequences can be presented at rates exceeding 2 dichotic word pairs/sec.

A few general comments concerning equipment may be useful. In speech generation, 16-bit computers are preferable to 12-bit computers. Also, fast cycle time is a significant factor since this influences maximum sampling rates, and, in particular, the amount of programming that the computer can execute between samples. Second, ADCs and DACs should have at least 6-bit (preferably 8-bit) resolution. Third, access time from mass storage is the major limiting factor in word presentation rate. With disks, rotation speed is a primary determinant of access time and high rotation speeds are advantageous. Fixed-head, rather than moving-head, disks generally provide faster access times.

\section{USEFUL TECHNIQUES AND PROCEDURES}

\section{Efficient Use of Available Memory}

To reproduce speech with quality similar to that heard on the telephone, speech frequencies up to $3,000 \mathrm{~Hz}$ must be maintained; for quality comparable to $\mathrm{AM}$ radio, frequencies up to $7,500 \mathrm{~Hz}$ are needed. These criteria require sampling rates of 6,000 and 15,000 samples/sec, respectively. Thus, if only one sample is stored in each available location in a $4 \mathrm{~K}$ memory, only very short speech segments can be retained. However, by packing two samples in each location, reasonably long speech segments can be retained, since computer memory is effectively doubled. With 16-bit computers, this means that each stored sample would consist of 8 bits of information. Since only 8-bit samples are needed for very high-quality speech, this sample-packing technique is ideal for 16-bit computers.

With 12-bit computers, each sample is limited to 6 bits. While reducing the number of bits per sample decreases speech quality (just as does reduced sampling rate), the effects of reduced sampling rates are more severe than the effects of reduced bits per sample. Roughly speaking, for an equivalent gain in storage space, reduction in bits per sample is preferable to reduction in sampling rate until less than 5 bits/sample are taken. Except for critical psychophysical applications, the gain in signal quality obtainable with more than 8-bit samples is negligible.

Improved information storage can also be achieved by more efficient digitization of the analog speech signal. Encoding efficiency is improved by considering characteristics of the human auditory system, in particular, the size of a just-detectable change in signal amplitude is proportional to the signal amplitude. Using this property, more information can be packed into a fixed number of bits by nonlinear encoding than by the linear encoding process characteristic of most ADCs. Linear encoding means that a 1-bit increment always represents the same increase in voltage, which is not so with nonlinear encoding. Since the human ear is sensitive to very small changes in the amplitude of small signals, but only to rather large changes in high amplitude signals, the appropriate nonlinear encoding system is one in which a 1-bit increment signifies a smaller voltage change for momentary low signal voltages than for momentary high signal voltages. Thus, with optimum nonlinearity (i.e., properly matched to the human auditory system), 7 bits/sample can be almost as effective as an 11-bit linearly encoded sample (e.g., Pierce, 1961). Nonlinear encoding can be achieved by altering the $\mathrm{ADCs}$ or introducing appropriate nonlinearities into the speech signal before it is presented to a linear ADC. The latter approach is generally more convenient. In our system, the input speech signal is fed through a logarithmic amplitude compression circuit. This reduces the amplitude of strong signals more than weak signals, providing the appropriate form of nonlinearity. At presentation, the nonlinearities are removed from the signal by applying an inverse transformation (amplitude expansion) to the signal that appears at the DAC outputs.

\section{Control of Stimulus Amplitude}

Control of speech amplitude is a difficult problem. Usually the goal has been to somehow equate signals in terms of overall loudness. However, there does not seem to be a simple speech parameter that can be selected as the preferred indicant of loudness. For one thing, the distribution and duration of momentary amplitude peaks and lulls may be as important as the average loudness over a long time period. At any rate, at least three approaches have been tried. First, at tempts are usually made to maintain constant signal loudness at input. The indicant of loudness is usually a calibrated VU meter with words spoken by a trained phonetician. Unfortunately, this does not insure that the output speech signal will be within amplitude tolerance since computer processing of speech characteristics such as 
duration may alter loudness by deleting or expanding amplitude peaks, for example.

A second method is to control the speech signal at time of presentation. One possibility is to compress the dynamic amplitude range of the output speech signal by amplifying weak signals and attenuating strong signals. Simple one-chip circuits to perform this function are available. While this method, combined with input control, helps maintain uniform loudness, the problem is not solved entirely. In addition, these methods provide no way of producing variations in signal loudness.

A newer approach in our laboratory has been to employ computer control of output speech amplitude. Prior to word presentation, the computer determines the variance (average of the squared voltage samples in the digitized representation, taking into account any nonlinear signal changes to be introduced subsequent to the DAC outputs) of the to-be-presented word. The computer then determines a gain factor so that, when a sample is multiplied by this factor, an experimenterspecified variance is obtained. This constant is used to control the gain of a linear amplifier connected to the DAC outputs (subsequent to any amplitude expansion circuitry, as mentioned previously). Linear amplifier or multiplier chips are available at modest cost (e.g., \$30). By specifying a desired variance, the experimenter can regulate stimulus loudness as it is related to this physical parameter.

\section{Presentation Rate}

When presenting sequences of words under minicomputer control, the main presentation rate limitation results from the time needed to retrieve digitized words stored on magnetic tape or disk. With on-line presentation, a word-relocation technique minimizes access time. Initially, digitized words are stored at various locations of the mass storage device. With word relocation, a block of space on the storage device is reserved for words that will be presented within the next word sequence. Words are retrieved from their haphazard locations all over the storage device and then loaded into the reserved block in the order in which they are needed. This eliminates most of the access time problem (especially with magnetic tapes). But this method increases intertrial delays by the time needed to relocate the word representations in the reserved mass-storage area.

For off-line applications a second method for reducing interword delays is available. With this method (Poltrock \& Mathews, 1976), the rate at which successive digitized samples are presented is one-half or onefourth the rate at which samples were taken. Thus, it takes twice or four times as long as normal to present a word. But, access and interword times are unchanged. A master tape containing abnormally slow word presentations can be recorded at, for example, 1-3/8 ips. Then, by playing the tape at $3-3 / 4$ or $7-1 / 2 \mathrm{ips}$, the words will be restored to their normal frequency range, while the interword intervals will be reduced by a factor of two or four. Unfortunately, this procedure cannot be extended indefinitely to further reduce interword intervals, since the frequency range of the slowed word representations on the master tape drops below the range of even the best tape recorders.

\section{Word-Length Adjustment}

In many applications it is important to control the duration of speech stimuli. For practical purposes, the computer is essential for this purpose. Two general methods have been developed for word-length adjustment. In both methods an estimate of the duration of the unprocessed word is made by the computer operator when the digitized representation is first obtained. The computer then compares this estimate with the desired word length specified by the computer operator. In the first method (Knight \& Kantowitz, 1973), the computer then deletes or repeats an entire medial section of the digitized word to produce the necessary shortening or expansion. The operator can vary the point at which the deletion or repetition begins. A disadvantage of this method is that important word features are often deleted, producing "SAKE" from the input word "STEAK." Consequently, adjustment of the cut or repetition starting point is critical and a rather limited range of length adjustment is possible.

With a second, new method (subroutine PROCRUSTES II), the computer distributes small deletions or repetitions throughout the entire word. Spacing these adjustments is done by computer so that they occur more than $1 / 30 \mathrm{sec}$ apart, thus avoiding introduction of spurious low-frequency tones into the speech signal. This procedure results in a greater range of possible word-length adjustments, although it is slower, and the necessary software consumes more memory. The adjustment is nearly always done without operator intervention, thus speeding the word-processing task.

Finally, in earlier versions of our speech-processing system, word-length adjustment was accomplished before storing words on the mass-storage device. This meant that a new digitized word representation had to be obtained each time a different word length was needed. At present, unprocessed digitized representations are stored on a disk along with parameters specifying unprocessed word lengths and amplitudes. These parameters then control adjustment at time of presentation according to experimenter specifications.

\section{System Software}

Early versions of our speech-processing system software were compact, integrated, single programs, with as many built-in features as we could think of when the programs were being written. Unfortunately, new applications for which the programs were unsuitable seemed to present themselves as soon as the program was 
debugged. We have altered our software strategy toward production of independent special-purpose subroutines that can be strung together for any particular application. For example, while the early system could generate precisely aligned dichotic word pairs, it could not produce dichotic word pairs with any asynchrony. In the newer system, the programmer simply calls a sequence of three subroutines; the first presents one word on the right (for instance) for a short time, the next subroutine presents words on both left and right channels, and this is followed by a subroutine presenting a single word on the left. Finally, another aspect of our software strategy has been to minimize reliance on specific pieces of hardware. For example, software clocks replace the need for external hardware clocks.

\section{REFERENCES}

Cohen, M. M., \& Massaro, D. W. Real-time speech synthesis. Behavior Research Methods \& Instrumentation, $1976,8,189-196$.

KNIGHT, J. L., JR., \& KANTowitz, B. H. A minicomputer method for generating dichotic word pairs. Behavior Research Methods \& Instrumentation, 1973, 5, 231-234.

Pierce, J. R. Symbols, signals, and noise. New York: Harper \& Row, 1961.

Poltrock, S. E., \& Mathews, N. M. A system for computer control of auditory stimuli. Behavior Research Methods \& Instrumentation, 1976, 8, 197-199. 